\title{
Patient-reported outcomes among patients using exenatide twice daily or insulin in clinical practice in six European countries: the CHOICE prospective observational study
}

Matthew Reaney ${ }^{1}$, Chantal Mathieu ${ }^{2}$ Claes-Göran Östenson ${ }^{3}$, Stephan Matthaei ${ }^{4}$, Thure Krarup ${ }^{5}$, Jacek Kiljański ${ }^{6}$, Carole Salaun-Martin ${ }^{7}$, Hélène Sapin ${ }^{7}$, Michael Theodorakis ${ }^{8^{*}}$ and Bruno Guerci ${ }^{9}$

\begin{abstract}
Background: Improvements in the clinical condition of patients with type 2 diabetes are often accompanied by improvements in health-related quality of life and other patient-reported outcomes (PROs), but data assessing injectable treatment initiation from the patient's perspective in routine clinical practice are lacking. We examined PROs in patients initiating injectable treatment in the CHOICE (CHanges to treatment and Outcomes in patients with type 2 diabetes initiating InjeCtablE therapy) study.
\end{abstract}

Methods: CHOICE was a 24-month, prospective observational study conducted in six European countries. Patients initiated exenatide twice daily (BID) or insulin based on a physician's clinical judgement. Clinical and PRO data were collected at baseline (injectable therapy initiation) and after approximately 3, 6, 12, 18 and 24 months. The two treatment cohorts had different baseline characteristics; therefore, no statistical comparisons of endpoints between main cohorts were conducted.

Results: There were 2388 patients eligible for analysis (exenatide BID cohort, $n=1114$; insulin cohort, $n=1274$ ). Mean positive changes in Impact of Weight on Quality of Life-Lite (IWQOL-Lite) total score and EuroQoL5-Dimension (EQ-5D) index and visual analogue scale (VAS) scores were observed in both cohorts with most changes observed during the first 6 months after injectable therapy initiation. Patients who experienced weight loss ( $\geq 1 \mathrm{~kg}$ ) at 24 months appeared to have higher mean improvements in IWQOL-Lite total score than did patients with weight gain or no weight change. Patients who met the composite clinical endpoint of glycated haemoglobin ( $\mathrm{HbA} 1 \mathrm{c})<7.0 \%$, no weight gain $(\leq 1 \mathrm{~kg})$ and no hypoglycaemia generally experienced higher mean improvements in EQ-5D index and VAS scores (compared with patients who did not meet this endpoint) and Diabetes Health Profile-18 scores (versus the main cohorts). High levels of missing data were observed for all PRO measures in both cohorts compared with those for clinical outcomes.

Conclusions: These data from a clinical practice study support those from clinical trials, suggesting that PROs are not adversely affected, and may be improved, by injectable therapy initiation. PRO data may aid appropriate treatment selection for individual patients.

Trial registration: ClinicalTrials.gov, NCT00635492

Keywords: Diabetes mellitus, Type 2, Exenatide, Insulin, Injectable therapy, Patient-reported outcomes

\footnotetext{
*Correspondence: mtheod@ebie.gr

${ }^{8}$ Department of Clinical Therapeutics, University of Athens School of

Medicine, Athens, Greece

Full list of author information is available at the end of the article
} 


\section{Background}

The increasing global prevalence of type 2 diabetes (T2DM) is accompanied by increased clinical and economic burden [1]. Achieving good metabolic control, including tight control of blood glucose, contributes to reducing the clinical, psychological, and economic burden of T2DM, and this requires that healthcare professionals and patients work together to achieve optimal treatment of this chronic disease [2-5]. The influence of physical and social factors on T2DM incidence and health outcomes is also receiving attention [6]. Patients with T2DM require systematic, individualised and progressive interventions involving different therapies that address the clinical and psychosocial aspects of their illness [2,3,6,7]. A comprehensive evaluation of healthcare should ascertain a patient's expressed health needs [7], as patient understanding, engagement, and commitment to the prescribed treatment strategy is key to meeting treatment goals and reducing morbidity and mortality associated with T2DM [3]. A patient's perception of how his or her condition or treatment affects his or her quality of life is an important consideration when making treatment decisions, and physicians should consider this information as well as clinical data when discussing the available options with their patients $[3,8]$.

The health-related quality of life (HRQoL) of patients with diabetes is often impaired, compared with a population without diabetes, and can be affected by both clinical and psychosocial factors $[8,9]$. HRQoL is inversely correlated with diabetes severity $[5,9,10]$, and improvements in the clinical condition of patients with diabetes, particularly T2DM, are often accompanied by improvements in HRQoL and some other related patient-reported outcomes (PROs), such as health status and psychological well-being [8,11-14]. Newer T2DM therapies such as glucagon-like peptide-1 (GLP-1) receptor agonists are typically associated with weight loss; these therapies have been associated with improvements in weight-related quality of life (i.e. quality of life pertaining to weight) [11] and may also demonstrate other benefits on relevant PROs. Improvements in PROs have been reported from several randomised clinical trials (RCTs) of exenatide twice daily (BID) [15-17]. Although RCTs provide valuable data regarding the efficacy of a drug in an 'ideal' setting, the populations and management approach used in RCTs may not reflect actual clinical practice, where treatment is more complex and diverse [18]. Prospective observational studies are therefore used to investigate effectiveness, i.e., how well drugs work under real world conditions subject to several sources of variation, including patient characteristics, comorbidities and concomitant medications. Naturalistic studies such as these, conducted with less structure than RCTs, for a longer duration of time, and with a larger sample size, may yield different findings [17] and enhance the evidence upon which the management of T2DM is based $[3,19,20]$.

The aim of the analyses reported in this manuscript was to understand the patient's perspective following initiation of injectable antidiabetes medication in routine clinical practice. PROs were examined using data from the CHOICE (CHanges to treatment and Outcomes in patients with type 2 diabetes initiating InjeCtablE therapy) study [21-24]. Exenatide BID and insulins were the only injectable treatments available for T2DM when this study commenced. Therefore, the study recruited patients initiating either exenatide BID or their first insulin regimen in routine clinical practice. Baseline patient characteristics [21] and clinical outcomes, healthcare resource use, and costs during the 24 months after initiation of injectable therapy in CHOICE have been reported elsewhere [22-24]. Understanding PROs following injectable therapy initiation will provide additional insight from the patient's perspective that, together with clinical data, will help patients and clinicians to make better informed treatment decisions.

\section{Patients and methods Study design and patients}

CHOICE (www.clinicaltrials.gov identifier NCT00635492) was a prospective, noninterventional observational study that recruited patients from six European countries (Belgium, Denmark, France, Germany, Greece, and Sweden) between January 2008 and October 2009. Patients aged $\geq 18$ years and initiating their first injectable antidiabetes therapy with exenatide BID or insulin for T2DM in routine clinical practice were included in the study. Patients were invited to participate in CHOICE only after the clinical decision had been made to initiate exenatide BID or insulin for the treatment of T2DM (in addition to any oral antidiabetes drugs required). Treatment choice (exenatide BID or insulin) was based on the clinical judgement of the patient's physician. Patients gave written informed consent for the use of their data and appropriate ethical review board approval was obtained from the Ethics Committee of the State Medical Association (Frankfurt, Germany), the Regional Ethical Review Board (Stockholm, Sweden), and the Medical Ethics Committee of University Hospitals Leuven (Leuven, Belgium). Further details on the design of the CHOICE study have been reported previously [21].

The primary endpoint of CHOICE was the time from the initiation of initial injectable regimen (exenatide BID or insulin) to significant treatment change (see Mathieu et al. [22] for definitions). The study also aimed to describe the characteristics of patients with T2DM initiated on injectable therapy [21], the factors associated with changes to treatment, clinical outcomes, PROs, and the healthcare resource use observed over 24 months. 
Data were collected from each patient at baseline (initiation of injectable therapy) and at follow-up visits when they occurred as part of clinical practice, approximately $3,6,12,18$, and 24 months (all \pm 6 weeks) after baseline.

\section{Measures}

PRO endpoints were measured using standardised and validated questionnaires. Weight-related quality of life was assessed using the Impact of Weight on Quality of Life-Lite questionnaire (IWQOL-Lite), a 31-item scale that assesses the domains of physical function, selfesteem, sexual life, public distress, and work [25]. Response categories range from $1=$ "never true" to 5 = "always true". Total scores are transformed in a linear manner to IWQOL-Lite "standardised scores", ranging from 0 to 100 , with higher standardised scores indicating better quality of life [26].

Health status was measured using the generic EuroQol-5-Dimension (EQ-5D) instrument [27] [threelevel (3L) version]. In the EQ-5D, patients are asked to report their level of functioning in five dimensions (mobility, self-care, usual activities, pain/discomfort, anxiety/ depression), with each dimension assessed by one item with three response choices (no problems, some problems, severe problems). Responses to the five items are used to derive an overall health index score (using the UK weighting) with a possible range from -0.594 to 1.0 , where 0 represents death and 1.0 represents a perfect health state (values below zero represent a state considered to be "worse than death") [28]. In addition, the EQ-5D contains a single item visual analogue scale (VAS) on which patients rate their current health state on a scale ranging from 0 (worst imaginable health state) to 100 (best imaginable health state).

HRQoL was measured using the Diabetes Health Profile-18 instrument (DHP-18), an 18-item diabetesspecific questionnaire with three domains: barriers to activity, disinhibited eating, and psychological distress [29]. Each question is scored using a 4-point Likert-type scale ranging from 0 to 3 , and subscale raw scores can be transformed to a common score range of $0-100$ with 0 representing no dysfunction.

Emotional distress was measured using the Hospital Anxiety and Depression Scale (HADS), a 14-item questionnaire (seven items each for anxiety or depression) for which each item is answered on a four-point scale (0-3) [30]. Raw subscale scores for anxiety and depression are calculated by adding all item scores together for a maximum possible score of 21 . The developers provide clinically defined cut-off points to indicate whether a patient is "within the normal range" (score of 0-7), or in a "mildly" (8-10), "moderately" (11-14), or "severely" (15-21) disordered state.

\section{Analysis}

\section{Sample size justification}

The sample size for CHOICE was based on the primary endpoint of time to first significant treatment change [21,22]; as such the study was not powered to assess changes in PROs. Sample size was calculated using a Monte-Carlo simulation, assuming annual patient dropout rates of $10 \%$ to $15 \%$ and a median time to significant treatment change of 9.0 months for the exenatide BID cohort and 8.6 months for the insulin cohort $[31,32]$. Based on this, the study aimed to recruit a maximum of 800 patients per country/country group. The insulin cohort was to be larger than the exenatide BID cohort ( $60 \%$ vs. $40 \%$ of patients) because of the greater variability in the insulin cohort (linked to use of different insulin regimens).

\section{Statistical analysis}

All patients who provided consent to release information, fulfilled study entry criteria, had a case report form summary page signed by an investigator and had at least one post-baseline assessment were included in the analyses ("eligible patients"). Due to the observational nature of this study, patients who violated the study description or who discontinued early from the study were included in the analyses.

As anticipated, analysis of the baseline data indicated that the two treatment cohorts comprised substantially different patient populations [21]. As a consequence, statistical comparisons of endpoints between the two main cohorts were not conducted and analyses of PRO endpoints are descriptive only.

Analyses of the PRO endpoints were conducted using available data from all eligible patients; data collected until study discontinuation were analysed according to the cohort (exenatide BID or insulin) that patients were placed in at baseline ("initiators" analysis). Item, domain, and total scores were summarised, as relevant, using frequency distribution and descriptive statistics. Absolute numbers and percentages (based on the number of patients with visits at the respective time point, as these patients had the opportunity to provide data) were given for categorical variables. Patients in Germany were not asked to complete the HADS or IWQOL-Lite questionnaires (due to general ethical concerns in Germany that patients may potentially feel overburdened when asked to complete several questionnaires), so percentage data for these measures are based on the number of patients with visits, excluding those patients in Germany. Item-level missing data were dealt with according to the instructions from the PRO instrument developers. The potential relationship between various clinical parameters and relevant PROs was also examined, for example, whether IWQOL-Lite scores were associated with weight loss or gain. 
Cox regression models were performed post hoc to investigate the association of baseline characteristics with time to achieving the clinically relevant composite endpoint of $\mathrm{HbA} 1 \mathrm{c}<7.0 \%$, no weight gain $(\leq 1 \mathrm{~kg})$, and no hypoglycaemia [33].

\section{Interpretation of PRO data}

To assist in interpreting PRO scores, a meaningful change in individual patient scores needs to be identified [34,35]. The proportion of patients meeting minimally important changes (MICs) in individual PRO scores was determined using published recommendations where available. Therefore, a change from baseline of $>0.03$ on the EQ-5D index [36], a change of $>3.0$ on the EQ VAS [36], and change in DHP-18 scores for barriers to activities of $>5.29$, disinhibited eating of $>2.80$ and psychological distress of $>4.87$ [37] constituted a MIC.

As there are no published MICs for the HADS (except in patients with chronic obstructive pulmonary disease [38]) and those for the IWQOL-Lite [39] considered participants enrolled in weight loss studies/programmes only (it was not reported whether any participants had diabetes), the distributions of responses to these questionnaires were calculated using a cumulative distribution function (CDF). This shows all magnitudes of change across the entire study population and the proportion of patients at each point along the scale score continuum who experience change at that level or lower [40]. This allows the reader to calculate the percentage of responders at each value of the change score and evaluate the consistency of changes across different response thresholds.

\section{Results}

\section{Clinical endpoints in CHOICE}

A total of 2515 patients were recruited from 322 investigator sites, mainly secondary care sites. Overall, there were 2388 eligible patients in this analysis; 1114 in the exenatide BID cohort and 1274 in the insulin cohort. Visit attendance decreased over time to 873 exenatide BID (78.4\%) and 1025 insulin (80.5\%) patients at the 24-month visit. Over 24 months, $23.5 \%$ of patients discontinued the study: lost to follow-up was the primary reason in both cohorts (about 13.5\% of each cohort), although $7.4 \%$ of exenatide BID and $3.5 \%$ of insulin patients discontinued due to subject decision [22].

Significant differences were observed in the baseline patient characteristics of the exenatide BID and insulin treatment cohorts (see Matthaei et al. [21]). Statistical comparisons of clinical and PRO endpoints between the two main cohorts were therefore not conducted.

A total of 470 patients from the exenatide BID cohort (42.2\%) and 459 patients from the insulin cohort (36.0\%) had a significant treatment change [22] during the study. In the exenatide BID cohort, $74.3 \%$ of the first significant treatment changes were discontinuations of initial injectable therapy, with the rest comprising the addition of oral or injectable antidiabetes medication to their exenatide BID regimen. Overall, the most common first significant treatment change for insulin patients was the addition of a new oral or injectable medication $(58.2 \%$ of first significant treatment changes). Discontinuations of $\geq 1$ insulin initiated at baseline accounted for $24.2 \%$ of the first significant treatment changes for patients in the insulin cohort.

During the study, 393 patients in the exenatide BID cohort (35.3\%) and 155 patients in the insulin cohort (12.2\%) discontinued their initial injectable therapy. The most common reason for such discontinuation in both cohorts was inadequate response [170 patients in the exenatide BID cohort (15.3\%) and 87 patients in the insulin cohort (6.8\%)]. Adverse events were cited as the reason for treatment discontinuation for 91 patients in the exenatide BID cohort $(8.2 \%)$ and 11 patients in the insulin cohort (0.9\%) [22].

Table 1 presents the main clinical data at baseline and 24 months and a full description of the clinical data has been reported previously [22]. Glycaemic control improved in both the exenatide BID and insulin cohorts. A mean weight loss was seen in the exenatide BID cohort, whereas a mean weight gain was seen in the insulin cohort. Gastrointestinal (GI) events were experienced by $30.8 \%$ of the exenatide BID cohort and $5.3 \%$ of the insulin cohort. The proportion of exenatide BID patients with GI events was higher in the first 6 months of the study (26.2\% of patients with data) than in subsequent 6 -month periods ( $<8 \%$ of patients with data).

\section{Patient-reported outcomes}

Table 2 summarises the baseline PRO scores for patients in the exenatide BID and insulin cohorts. High levels of missing data and large standard deviations (SDs) were noted for all PRO measures in both cohorts.

\section{IWQOL-Lite}

There was a mean positive change in overall IWQOLLite total score from baseline in both treatment cohorts during the first 6 months after initiation of injectable therapy (Figure 1a). Thereafter scores tended to plateau in the exenatide BID cohort and decrease in the insulin cohort, remaining above baseline levels throughout the 24-month study. When weight change $(\geq 1 \mathrm{~kg}$ in either direction) at 24 months was considered, mean (SD) baseline IWQOL-Lite scores for patients who later had weight change (compared with baseline) at 24 months were: exenatide BID, weight loss 75.51 (19.64); weight gain 79.17 (18.63); no weight change 80.48 (18.00); insulin: weight loss 81.17 (19.41); weight gain 85.09 (16.57); no weight change 85.81 (17.42). Mean (SD) changes in 
Table 1 Baseline characteristics and 24-month clinical outcomes: patients initiated on exenatide twice daily (BID) or insulin

\begin{tabular}{|c|c|c|c|c|}
\hline \multirow[t]{3}{*}{ Variable } & \multicolumn{2}{|c|}{ Exenatide BID } & \multicolumn{2}{|c|}{ Insulin } \\
\hline & \multirow{2}{*}{\multicolumn{2}{|c|}{ Baseline }} & \multicolumn{2}{|c|}{ Baseline } \\
\hline & & & \multicolumn{2}{|c|}{$(\mathrm{N}=1274)^{\mathrm{a}}$} \\
\hline \multirow[t]{2}{*}{ Male, $n(\%)$} & \multicolumn{2}{|c|}{$n=1114$} & \multicolumn{2}{|c|}{$n=1274$} \\
\hline & \multicolumn{2}{|c|}{$598(53.7)$} & \multicolumn{2}{|c|}{$733(57.5)$} \\
\hline \multirow[t]{2}{*}{ Age, years } & \multicolumn{2}{|c|}{$\mathrm{n}=1114$} & \multicolumn{2}{|c|}{$n=1274$} \\
\hline & \multicolumn{2}{|c|}{$58.1(10.1)$} & \multicolumn{2}{|c|}{$63.7(10.9)$} \\
\hline \multirow[t]{2}{*}{ Time since diabetes diagnosis, years } & \multicolumn{2}{|c|}{$n=1105$} & \multicolumn{2}{|c|}{$n=1263$} \\
\hline & \multicolumn{2}{|c|}{$8.2(5.7)$} & \multicolumn{2}{|c|}{$9.8(7.3)$} \\
\hline Diabetes complications, $n$ (\%) & \multicolumn{2}{|c|}{$n=1114$} & \multicolumn{2}{|c|}{$n=1274$} \\
\hline$\geq 1$ macrovascular complication & \multicolumn{2}{|c|}{$200(18.0)$} & \multicolumn{2}{|c|}{$320(25.1)$} \\
\hline \multirow[t]{3}{*}{$\geq 1$ microvascular complication } & & & & \\
\hline & Baseline & 24 months & Baseline & 24 months \\
\hline & $(\mathrm{N}=1114)^{\mathrm{a}}$ & $(\mathrm{N}=873)^{\mathrm{a}}$ & $(N=1274)^{a}$ & $(\mathrm{~N}=1025)^{\mathrm{a}}$ \\
\hline Weight, kg & $n=1112$ & $n=810$ & $n=1270$ & $n=947$ \\
\hline & $101.2(21.7)$ & $98.3(21.3)$ & $84.2(17.6)$ & $86.7(17.8)$ \\
\hline $\mathrm{BMI}, \mathrm{kg} / \mathrm{m}^{2}$ & $n=1100$ & $n=805$ & $n=1265$ & $n=942$ \\
\hline & $35.3(6.6)$ & $34.2(6.4)$ & $29.7(5.4)$ & $30.6(5.5)$ \\
\hline Blood pressure, $\mathrm{mmHg}$ & $n=1103$ & $n=769$ & $n=1259$ & $n=895$ \\
\hline Systolic & $137.7(16.5)$ & $134.8(15.2)$ & $137.4(17.4)$ & $133.9(15.3)$ \\
\hline Diastolic & $81.7(9.6)$ & $78.7(9.7)$ & $80.2(9.9)$ & $78.0(8.8)$ \\
\hline $\mathrm{HbA1c}, \%$ & $n=1087$ & $n=812$ & $\mathrm{n}=1245$ & $n=944$ \\
\hline & $8.4(1.4)^{b}$ & $7.3(1.2)$ & $9.2(1.9)^{b}$ & $7.3(1.1)$ \\
\hline Patients with $\mathrm{HbA} 1 \mathrm{c}<7 \%, n(\%)$ & $n=1087$ & $n=705$ & $n=1245$ & $\mathrm{n}=871$ \\
\hline & $128(11.8)$ & $258(36.6)^{c}$ & $75(6.0)$ & $333(38.2)^{c}$ \\
\hline No. of OADs used, $n(\%)$ & $n=1114$ & $n=873$ & $n=1274$ & $n=1025$ \\
\hline 0 & $76(6.8)$ & $94(10.8)$ & $333(26.1)$ & $304(29.7)$ \\
\hline 1 & $499(44.8)$ & $375(43.0)$ & $574(45.1)$ & $473(46.1)$ \\
\hline 2 & $491(44.1)$ & $341(39.1)$ & $341(26.8)$ & $220(21.5)$ \\
\hline$\geq 3$ & $48(4.3)$ & $63(7.2)$ & $26(2.0)$ & $28(2.7)$ \\
\hline Patients with $\geq 1$ hypoglycaemic event, $n(\%)^{d}$ & $n=1112$ & $n=1061$ & $n=1274$ & $n=1221$ \\
\hline & $59(5.3)$ & $195(18.4)$ & $56(4.4)$ & 449 (36.8) \\
\hline Patients with $\geq 1$ Gl symptom, $n$ (\%) & $n=1113$ & $n=1060$ & $n=1273$ & $n=1219$ \\
\hline & $72(6.5)$ & $327(30.8)^{\mathrm{e}}$ & $47(3.7)$ & $64(5.3)^{\mathrm{e}}$ \\
\hline Patients achieving lipid targets, $n(\%)$ & & & & \\
\hline $\mathrm{HDL}-\mathrm{C}>50 \mathrm{mg} / \mathrm{dl}$ & $n=989$ & $n=651$ & $n=1083$ & $n=737$ \\
\hline & $287(29.0)$ & $234(35.9)$ & $336(31.0)$ & $278(37.7)$ \\
\hline LDL-C <100 mg/dl & $n=967$ & $n=635$ & $n=1055$ & $n=729$ \\
\hline & $420(43.4)$ & $292(46.0)$ & $384(36.4)$ & $307(42.1)$ \\
\hline
\end{tabular}


Table 1 Baseline characteristics and 24-month clinical outcomes: patients initiated on exenatide twice daily (BID) or insulin (Continued)

\begin{tabular}{lcccc}
\hline Triglycerides $<150 \mathrm{mg} / \mathrm{dl}$ & $\mathrm{n}=1005$ & $\mathrm{n}=659$ & $\mathrm{n}=1118$ & $\mathrm{n}=753$ \\
& $362(36.0)$ & $326(49.5)$ & $450(40.3)$ & $\mathrm{n}=1113(55.4)$ \\
$\mathrm{HDL}-\mathrm{C}>50, \mathrm{LDL}-\mathrm{C}<100$ \& Triglycerides $<150 \mathrm{mg} / \mathrm{dl}$ & $\mathrm{n}=1012$ & $\mathrm{n}=659$ & $\mathrm{n}=748$ \\
& $73(7.2)$ & $81(12.3)$ & $75(6.7)$ & $82(11.0)$ \\
\hline
\end{tabular}

Continuous data are means (SD).

$\mathrm{BID}=$ twice daily; $\mathrm{BMI}=$ body mass index; $\mathrm{HbA1c}=$ glycated haemoglobin; $\mathrm{OAD}=$ oral antidiabetes drug; $\mathrm{Gl}=$ gastrointestinal; $\mathrm{HDL}-\mathrm{C}=$ high-density lipoprotein cholesterol; LDL-C = low-density lipoprotein cholesterol; SD = standard deviation.

${ }^{a} \mathrm{~N}$ numbers represent the number of patients who attended a baseline or 24-month visit. Percentages are based on the number of patients with data.

${ }^{\mathrm{b}}$ Most recent in previous 3 months.

'Data from the subgroup of 959 patients in the exenatide BID initiators group and 1170 patients in the insulin initiators group with baseline HbA1C $\geq 7.0 \%$ at baseline (254 and 299 patients, respectively, had missing data).

dPatient recall: baseline = past 3 months; 24 months = patients recalled events at each visit (between baseline and 24 months) that they had experienced since their previous visit.

${ }^{\text {e}}$ Patient recall: Baseline = past 4 weeks; 24 months = patients recalled symptoms at each visit (between baseline and 24 months) that they had experienced since their previous visit.

IWQOL-Lite scores (according to weight change) from baseline to 24 months in the exenatide BID cohort were: weight loss +4.36 (13.90), weight gain -0.13 (12.34), no weight change +1.18 (13.32). Respective change values for the insulin cohort were: +2.98 (13.16), -0.04 (11.57), +1.61 (11.01).

The CDF for the IWQOL-Lite total score (Figure 2) showed that $57.7 \%$ of exenatide BID and $53.0 \%$ of insulin patients reported no worsening in IWQOL-Lite scores at the 24-month visit (missing data overall: 13.6\%).

\section{$E Q-5 D$}

The proportions of patients with visits (in both cohorts) who reported at least some problems with the single domains "mobility", "self-care" and "usual activities" of the EQ-5D generally showed little change over the 24 months of the study (data not shown), although the percentage of patients overall with missing data for EQ-5D single domains increased from $\sim 2 \%$ at baseline to $\sim 12 \%$ at the 24 -month visit. However, the proportions of patients reporting at least some problems with "pain/discomfort" were $52.8 \%$ at baseline and $43.4 \%$ at 24 months in the exenatide BID cohort and $53.3 \%$ at baseline and $47.4 \%$ at 24 months in the insulin cohort. The proportions of patients who reported at least some problems with "anxiety/ depression" were $46.9 \%$ at baseline and $35.4 \%$ at 24 months in the exenatide BID cohort; respective values were $47.6 \%$ and $35.9 \%$ in the insulin cohort.

The greatest mean improvements in the EQ-5D index score were observed between baseline and 6 months for both cohorts (Figure 1b). Mean changes were above MIC for the exenatide BID cohort at all time points, and were above MIC only at 6 months in the insulin cohort; between baseline and 24 months, $32.0 \%$ of patients in the exenatide BID cohort and $27.3 \%$ of patients in the insulin cohort had improved EQ-5D index scores by more than the MIC (Table 3). Similarly, both cohorts experienced a mean increase in the EQ-VAS score over
24 months, with most change occurring between baseline and 6 months (Figure 1c). Mean changes in EQ-VAS scores were above the MIC at all time points for both cohorts; EQ-VAS scores had improved by more than the MIC for $47.4 \%$ of patients in the exenatide BID cohort and $44.7 \%$ of patients in the insulin cohort at 24 months (Table 3). Post hoc multivariate Cox regression models showed that baseline EQ-5D index values (VAS scores were not evaluated) were not significantly associated with time to achieving the composite endpoint proposed by Zinman et al. [33]. However, patients in both the exenatide BID and insulin cohorts who met the composite endpoint ( $\mathrm{n}=271$ in the exenatide BID cohort, $\mathrm{n}=144$ in the insulin cohort) experienced numerically greater changes in mean (SD) EQ-5D index and VAS score after 24 months [exenatide BID: index: $+0.08(0.23)$, VAS: +9.01 (18.24); insulin: index: +0.02 (0.32), VAS: +7.57 (21.78)] than the respective group of patients who did not meet the composite endpoint [exenatide BID: index: +0.04 (0.24), VAS: +6.69 (16.69); insulin: index: +0.01 (0.26), VAS: +4.63 (16.95)].

\section{DHP-18}

Changes in DHP-18 scores over the 24-month study are presented in Figure 1d. Most changes were small and below their respective MIC, except for change in disinhibited eating at all time points for the exenatide BID cohort. The number of patients whose DHP-18 scores improved or worsened by more than the MIC at the 24-month visit is shown in Table 3.

In both cohorts, patients who met the composite endpoint had numerically lower (better) mean baseline scores compared with their respective total cohort for all DHP-18 parameters (data not shown; baseline scores for the total cohort are presented in Table 2). Patients who met the composite endpoint also generally experienced numerically greater DHP- 18 score improvements over 24 months than those in the main cohort. The differences in DHP-18 
Table 2 Patient-reported outcome (PRO) scores at baseline

\begin{tabular}{|c|c|c|}
\hline PRO & $\begin{array}{l}\text { Exenatide BID } \\
\qquad \mathrm{N}=1114^{\mathrm{a}}\end{array}$ & $\begin{array}{c}\text { Insulin } \\
N=1274^{a}\end{array}$ \\
\hline \multicolumn{3}{|l|}{ IWQOL-Lite* } \\
\hline \multirow[t]{2}{*}{ Total score } & $n=715$ & $n=817$ \\
\hline & $77.25(19.41)$ & $84.49(17.37)$ \\
\hline Subscales & $n=648-707^{b}$ & $\mathrm{n}=725-812^{\mathrm{b}}$ \\
\hline Physical function & $69.35(23.68)$ & $77.84(21.03)$ \\
\hline Self-esteem & $76.31(26.68)$ & $86.47(21.41)$ \\
\hline Sexual life & $76.25(28.50)$ & $82.54(26.81)$ \\
\hline Public distress & $89.25(18.77)$ & $93.60(15.51)$ \\
\hline Work & $85.50(20.15)$ & $90.25(18.09)$ \\
\hline \multicolumn{3}{|l|}{ EQ-5D } \\
\hline \multirow[t]{2}{*}{ Index score ${ }^{* *}$} & $n=1079$ & $n=1242$ \\
\hline & $0.73(0.27)$ & $0.71(0.27)$ \\
\hline Subscales & $n=1086-1090$ & $n=1249-1254$ \\
\hline \multicolumn{3}{|c|}{ Number $(\%)^{c}$ of patients who reported at least some problems with: } \\
\hline Mobility & $322(28.9)$ & $442(34.7)$ \\
\hline Self-care & $76(6.8)$ & $125(9.8)$ \\
\hline Usual activities & $232(20.8)$ & 301 (23.6) \\
\hline Pain/discomfort & $588(52.8)$ & $679(53.3)$ \\
\hline Anxiety/depression & $523(46.9)$ & $607(47.6)$ \\
\hline \multirow[t]{2}{*}{ VAS score++ } & $n=1063$ & $n=1225$ \\
\hline & $64.63(17.94)$ & $63.77(19.13)$ \\
\hline \multicolumn{3}{|l|}{ DHP-18*** } \\
\hline \multirow[t]{2}{*}{ Barriers to activity } & $n=1090$ & $n=1251$ \\
\hline & $30.95(21.41)$ & $29.45(19.41)$ \\
\hline \multirow[t]{2}{*}{ Disinhibited eating } & $n=1089$ & $n=1253$ \\
\hline & $45.88(21.75)$ & $38.35(21.65)$ \\
\hline \multirow[t]{2}{*}{ Psychological distress } & $n=1086$ & $n=1245$ \\
\hline & $29.18(21.34)$ & $26.53(21.07)$ \\
\hline \multirow[t]{2}{*}{$\mathrm{HADS}^{+}$anxiety } & $n=692$ & $n=794$ \\
\hline & $6.38(4.40)$ & $6.96(4.59)$ \\
\hline \multirow[t]{2}{*}{ HADS $^{+}$depression } & $n=695$ & $n=787$ \\
\hline & $5.44(4.09)$ & $6.04(4.35)$ \\
\hline
\end{tabular}

Data are presented as mean (SD), unless otherwise stated.

$\mathrm{PRO}=$ patient-reported outcome; BID = twice daily, IWQOL-Lite = Impact of Weight on Quality of Life-Lite, EQ-5D = EuroQoL-5D, VAS $=$ visual analogue scale DHP-18 = Diabetes Health Profile-18, HADS = Hospital Anxiety and Depression Scale.

${ }^{a} \mathrm{~N}$ represents the number of patients who attended a baseline visit. Percentages are based on N. The number of patients with data for each endpoint or group of endpoints $(\mathrm{n})$ is presented above that endpoint or group of endpoints.

${ }^{b}$ For both cohorts, the lowest $n$ number for the IWQOL-Lite subscales was for data for the sexual life subscale, the highest $n$ number was for the physical function subscale.

${ }^{\mathrm{C}}$ Percentages are based on the number of patients who attended the baseline visit.

*IWQOL-Lite standardised scores, range 0-100. Higher scores indicate higher quality of life. As IWQOL-Lite was not applied in Germany, the numbers of patients who attended a baseline visit and had the opportunity to provide IWQOL-Lite data were 730 (exenatide BID cohort) and 836 (insulin cohort).

**U.K.-specific coefficients and country-specific coefficients were used where available. Higher scores indicate better health status.

++ Range $0-100$. Higher scores indicate better health status.

***Standardised scores for subscales, range 0-100. Lower scores indicate better health-related quality of life.

+Raw scores for subscales, range 0-21. Lower scores indicate lower levels of emotional distress. As HADS Anxiety and Depression were not applied in Germany, the number of patients who attended a baseline visit and had the opportunity to provide HADS data were 730 (exenatide BID cohort) and 836 (insulin cohort). 


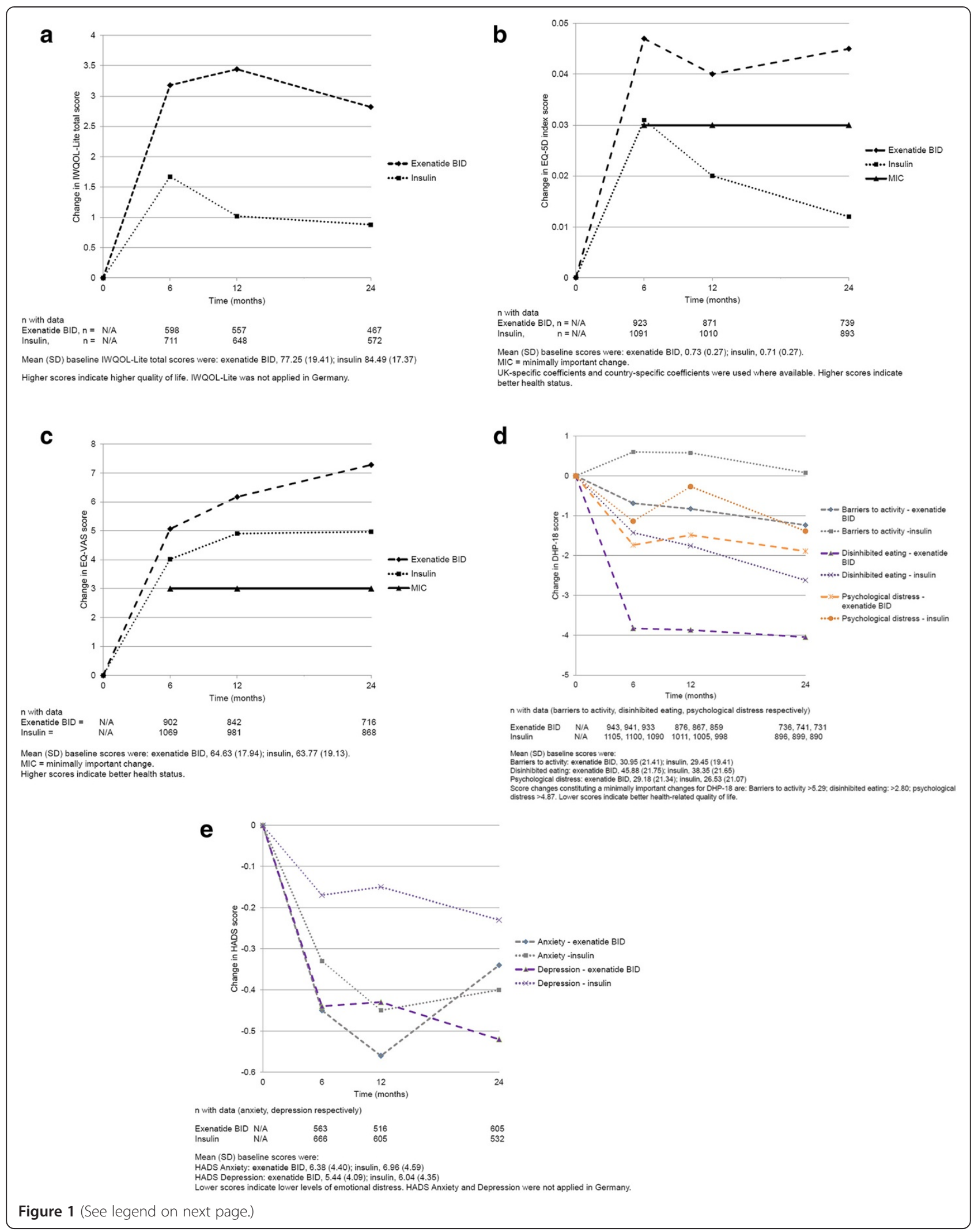


(See figure on previous page.)

Figure 1 Changes in patient-reported outcomes (PROs) over 24 months in the CHOICE study. Changes from baseline in PRO measures over 24 months after initiation of exenatide twice daily (BID) or insulin are presented. The number of patients with change data at each time point is presented below the time point. a) Mean change in standardised Impact of Weight on Quality of Life-Lite (IWQOL-Lite) total score. b) Mean change in EuroQoL-5-Dimension (EQ-5D) index score. c) Mean change in EuroQoL-visual analogue scale (EQ-VAS) score. d) Mean changes in standardised Diabetes Health Profile-18 (DHP-18) scores. e) Mean change in Hospital Anxiety and Depression Scale (HADS) scores.

score changes between patients who met the composite endpoint and the main cohorts were generally lower than the MIC, with the exception of disinhibited eating at 24 months in the exenatide BID cohort (data not shown).

\section{HADS anxiety and depression}

Changes in HADS anxiety and depression scores are presented in Figure 1e.

At baseline, 59.2\% of exenatide BID and 53.8\% of insulin patients had responses "within the normal range" for anxiety. Responses in the "mildly", "moderately" or "severely" disordered states were given by $18.2 \%, 13.2 \%$ and $4.2 \%$ of exenatide BID patients and 20.8\%, 14.6\%, and 5.7\% of insulin patients, respectively. For depression, $66.2 \%$ of exenatide BID and $60.6 \%$ insulin patients had responses "within the normal range". Responses in the "mildly", "moderately" or "severely" disordered states were given by $16.3 \%, 10.0 \%$ and $2.7 \%$ of exenatide BID patients and $17.9 \%, 11.8 \%$ and $3.7 \%$ of insulin patients. The proportion of patients with responses within the normal range and in each disordered state generally decreased over the course of the study (data not shown), but changes were small and the amount of missing data increased (overall: anxiety $5.1 \%$ at baseline, $16.0 \%$ at 24 months; depression $5.4 \%$ at baseline, $17.4 \%$ at 24 months).

The CDFs for the HADS anxiety and depression scores (Figures $3 \mathrm{a}$ and $\mathrm{b}$ ) indicate that $52.6 \%$ and $50.3 \%$ of exenatide BID patients and $52.9 \%$ and $48.0 \%$ of insulin patients reported no worsening in HADS anxiety and depression scores, respectively, at the 24-month visit (missing data overall: anxiety $19.6 \%$; depression $21.5 \%$ ).

\section{Discussion}

The initiation of injectable therapy may represent an important milestone for the patient from both a clinical and personal point of view: in addition to the knowledge that their condition has progressed to requiring injectable therapy, the patient may be fearful of injections and side effects. CHOICE measured PROs at, and during the 24 months following, initiation of injectable therapy with exenatide BID or insulin. The data suggest that initiation of injectable therapy with either exenatide BID or insulin does not adversely affect weight-related quality of life, health status, HRQoL, or emotional distress, and may

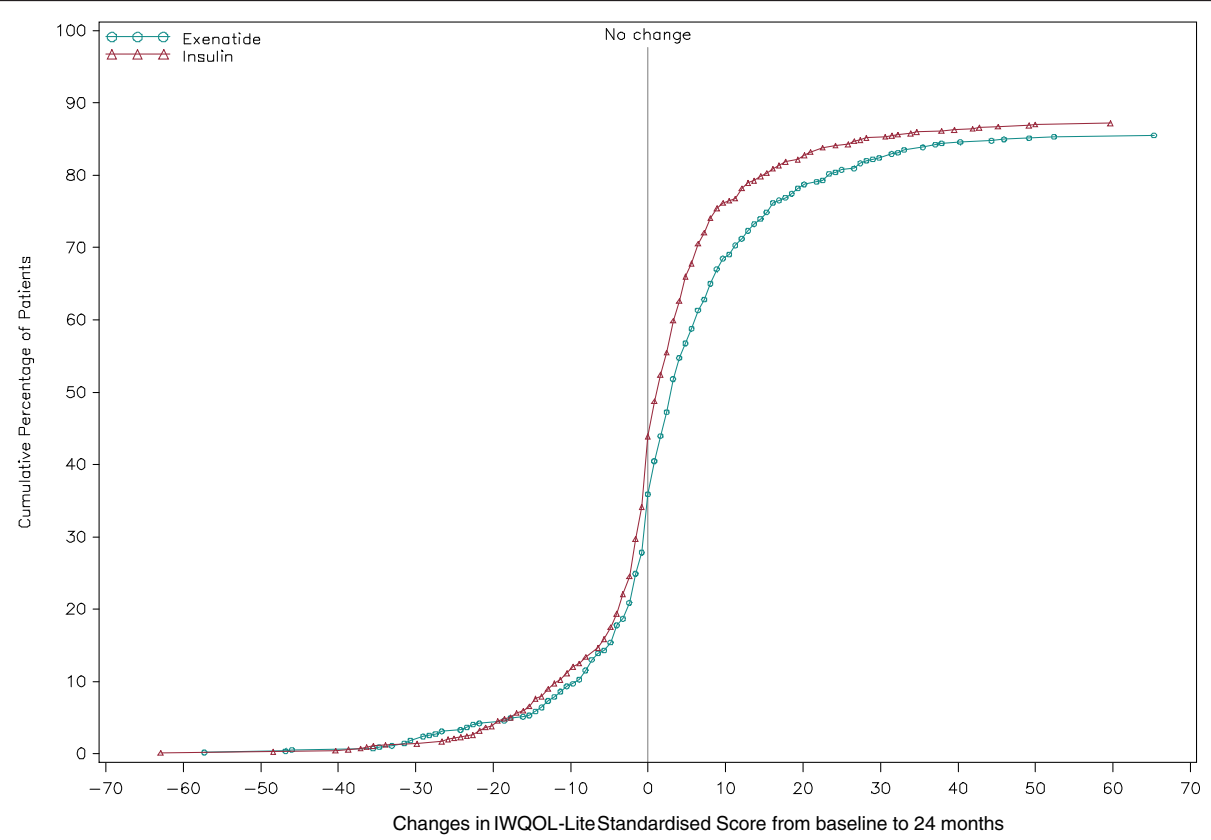

Figure 2 CDF of IWQoL-Lite questionnaire total score change from baseline to $\mathbf{2 4}$ months. CDF = cumulative distribution function; IWQOL-Lite = Impact of Weight on Quality of Life-Lite Higher scores indicate higher quality of life. IWQOL-Lite was not applied in Germany. 


\begin{tabular}{|c|c|c|}
\hline PRO & $\begin{array}{l}\text { Exenatide BID } \\
\qquad(\mathrm{N}=873)^{*}\end{array}$ & $\begin{array}{c}\text { Insulin } \\
(\mathrm{N}=1025)^{*}\end{array}$ \\
\hline EQ-5D index score & $n=739$ & $n=893$ \\
\hline n (\%) improving by > MIC (0.03) & $279(32.0)$ & $280(27.3)$ \\
\hline $\mathrm{n}(\%)$ worsening by $>$ MIC (0.03) & $194(22.2)$ & $288(28.1)$ \\
\hline EQ-5D VAS score & $n=716$ & $n=868$ \\
\hline n (\%) improving by > MIC (3.0) & $414(47.4)$ & $458(44.7)$ \\
\hline n (\%) worsening by > MIC (3.0) & $150(17.2)$ & $224(21.9)$ \\
\hline \multicolumn{3}{|l|}{ DHP-18 (standardised scores) } \\
\hline Barriers to activity & $n=736$ & $n=896$ \\
\hline n (\%) improving by > MIC (5.29) & $231(26.5)$ & $251(24.5)$ \\
\hline n (\%) worsening by > MIC (5.29) & $174(19.9)$ & $250(24.4)$ \\
\hline Disinhibited eating & $n=741$ & $n=899$ \\
\hline n (\%) improving by > MIC (2.80) & $364(41.7)$ & $415(40.5)$ \\
\hline n (\%) worsening by > MIC (2.80) & $247(28.3)$ & $308(30.0)$ \\
\hline Psychological distress & $n=731$ & $n=890$ \\
\hline n (\%) improving by > MIC (4.87) & $306(35.1)$ & $365(35.6)$ \\
\hline n (\%) worsening by > MIC (4.87) & $254(29.1)$ & $311(30.3)$ \\
\hline
\end{tabular}

$\mathrm{PRO}=$ patient-reported outcome; $\mathrm{MIC}$, minimally important change; $\mathrm{BID}=$ twice daily, EQ-5D = EuroQoL-5D, VAS = visual analogue scale, DHP-18 = Diabetes Health Profile-18.

${ }^{*} \mathrm{~N}$ numbers represent the number of patients who attended a 24-month visit. Percentages are based on $\mathrm{N}$. The number of patients with data for each endpoint $(n)$ is presented above each endpoint.

also be associated with improvements in some PRO measures. These PRO data support those from previous clinical trials [15-17] and help address an identified need for long-term prospective data to understand whether PRO benefits observed in trials of incretin therapy are realised in clinical practice [11]. Analysis of PRO data from the CHOICE study supports injectable therapy initiation, suggesting that, from the patients' perspective, the disutility associated with daily injections $[41,42]$ is offset by the clinical improvements observed. Consistent with clinical variables [22], most change in PRO measures was seen in the first 6 months.

Heavier body weight has been associated with disutility in patients with T2DM, and lower body weight with added utility [41]. In CHOICE, patients who later achieved weight loss $(\geq 1 \mathrm{~kg})$ appeared to have poorer weightrelated quality of life at baseline (i.e. before injectable therapy initiation), compared with those who experienced either no weight change or weight gain, suggesting that poor weight-related quality of life could improve motivation to lose weight. As expected, weight-related quality of life then appeared to be affected by whether or not patients gained or lost weight $(\geq 1 \mathrm{~kg})$ : those who experienced weight loss appeared to have higher mean IWQOL-Lite score changes than those with no weight change or weight gain, indicating an improvement in weight-related quality of life as well as the clinical benefits of weight loss for these patients. However, although patients in the exenatide BID cohort experienced a mean weight loss and those in the insulin cohort experienced a mean weight gain, an overall mean increase in IWQOL-Lite score was observed for both cohorts during the study. The improvement in IWQOL-Lite score following the initiation of exenatide BID in CHOICE is in agreement with 12-month results from the U.S. exenatide BID observational study (ExOS) [43].

EQ-5D index and VAS scores generally improved throughout the CHOICE study. These changes are encouraging, given that patients with T2DM in the longitudinal US Study to Help Improve Early evaluation and management of risk factors Leading to Diabetes (SHIELD) had a significantly greater decline in EQ-5D index scores over 5 years, compared with people without diabetes, and this decline was greater in patients with T2DM complications than in those without complications [44]. The proportion of patients in both cohorts of the CHOICE study reporting at least some problems with "anxiety/depression" numerically decreased over 24 months according to EQ-5D, and small mean improvements were observed in anxiety and depression according to the HADS. However, both these observations were confounded by increasing proportions of missing data from baseline to 24 months. At the 24-month visit, around half of patients in both cohorts reported no worsening in HADS anxiety and depression scores, although around $20 \%$ of patients had missing data for this analysis. Depression is a recognised problem in patients with T2DM [45], and both EQ-5D and HADS data may be important in monitoring this issue. EQ-5D data are required by reimbursement agencies to make decisions but HADS may be more relevant to clinical practice.

Although post hoc analyses revealed that baseline EQ-5D index score (VAS was not included in these analyses) was not significantly associated with time to achieving the composite clinical endpoint (HbA1c <7.0\%, no weight gain $(\leq 1 \mathrm{~kg})$, and no hypoglycaemia [33]), greater mean changes in EQ-5D index and VAS scores were observed for patients in both cohorts who met this composite endpoint during the study than for those who did not meet this endpoint. This suggests that achievement of meaningful clinical improvement (following injectable treatment initiation) may result in improved health status and that better health status at baseline may not influence the likelihood of patients later achieving meaningful clinical improvement.

All of the previously discussed PROs are generic and are used across a range of clinical conditions. Generic instruments include items that may be irrelevant and/or do not specifically enhance our understanding of the impact of diabetes, and they exclude domains that are likely to be of 


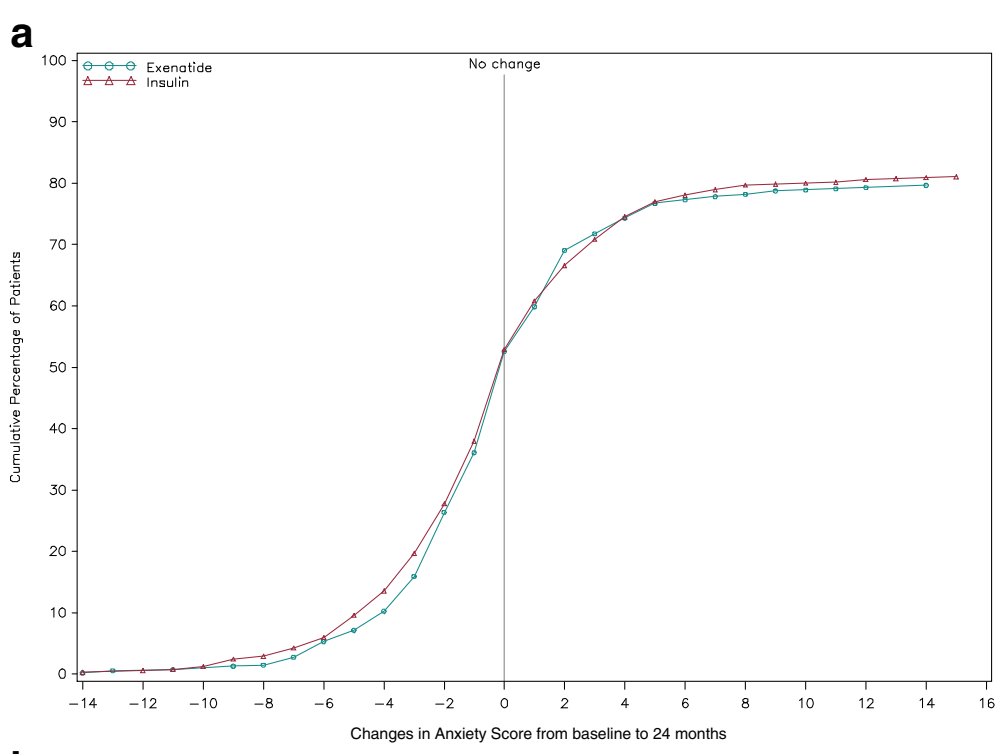

b

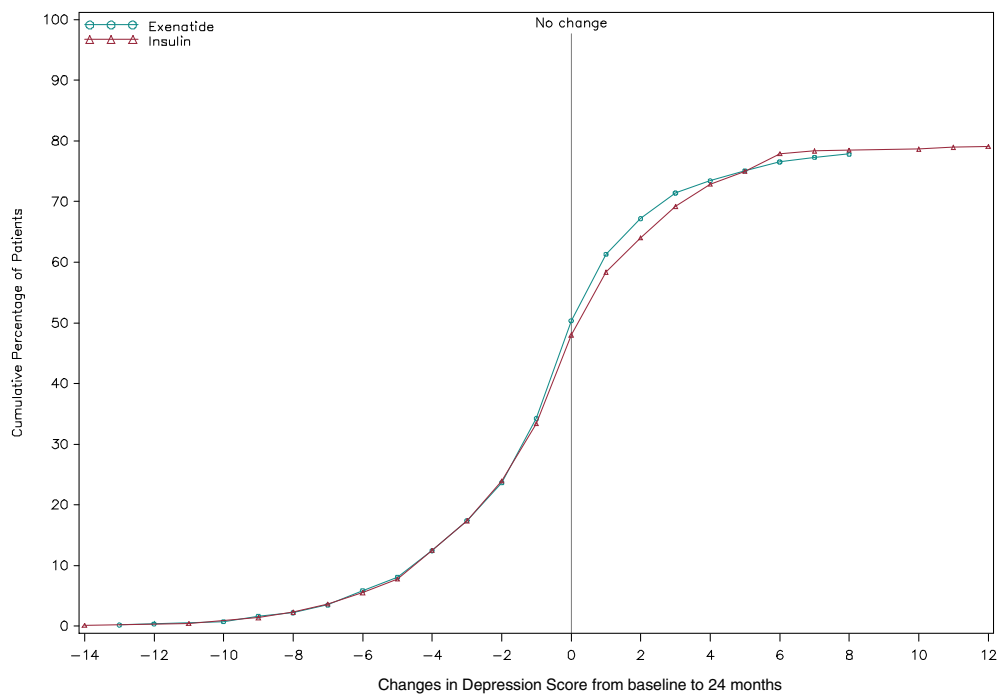

Figure $\mathbf{3}$ CDF of HADS score change from baseline to $\mathbf{2 4}$ months. a) CDF for HADS anxiety score change from baseline. b) CDF for HADS depression score change from baseline to 24 months. CDF = cumulative distribution function; HADS = Hospital Anxiety and Depression Scale Lower scores indicate lower levels of emotional distress. HADS Anxiety and Depression were not applied in Germany.

great relevance [46]. Diabetes-specific instruments do not allow comparison with other conditions, but they are likely to be "more sensitive to change and responsive to subgroup differences than a generic instrument" [47]. The DHP-18 is a diabetes-specific PRO measure and was used to examine the potential impact of injectable therapy initiation on diabetes-related HRQoL. Most changes in DHP-18 scores were below the relevant MICs, with the exception of improved disinhibited eating at all time points for the exenatide BID cohort. This improvement was achieved after 6 months and maintained throughout the study. It is interesting that the main improvement in disinhibited eating in the exenatide BID cohort was observed during the first 6 months. Although the proportion of exenatide BID patients experiencing GI events decreased as the study progressed, these data suggest that the improvement in disinhibited eating was not completely offset by GI events. Patients who met the composite clinical endpoint appeared to have higher DHP-18 score changes over 24 months than was observed in the total cohort.

These data add to a growing body of clinical evidence regarding the initiation of injectable therapies in patients with T2DM in routine clinical practice, by considering its potential impact on PROs. However, high levels of missing data were observed for PRO measures 
throughout the study compared with those observed for clinical outcomes [22]. There are a number of potential explanations for this. For example, PROs require the patient to complete questionnaires, and this may be burdensome to the patient [48], or considered as interventional or less important than obtaining clinical information if time is constrained. Indeed, two of the PRO questionnaires used in this study were not applied in Germany due to concerns about patient burden. This ethical constraint may have resulted in patients in Germany having a perceived lighter participation burden compared with patients in other countries. Patients may also be reluctant to answer certain questions that they consider to be too personal, especially if the setting lacks privacy. Indeed, for the IWQOL-Lite subscales in CHOICE, missing data were highest for the sexual life subscale. Additionally, the high level of missing data may itself be of significance. As patients generally comply with requirements for clinical data (e.g., give blood samples as requested), there is more scope for patients to choose whether or not to answer particular questions in questionnaires, and this may lead to a self-selection bias. For example, patients with a particularly high or low HRQoL may be more compelled to complete questionnaires.

In addition to the general limitations associated with prospective observational studies, this study has some further limitations. As physicians in routine practice likely chose exenatide BID or insulin based on different patient characteristics, the data for the two cohorts cannot be directly compared or attributed to either treatment, and no statistical comparisons of the cohorts were therefore performed. The analyses in CHOICE were based on an "initiators" analysis, in which patients remained in the cohort they were placed in at baseline regardless of whether or not they changed treatment, and no adjustment of PRO data for such changes was made. PROs may also be affected over time by cognitive reframing, a natural fluctuation that can result in changes in patients' perceptions of baseline feelings that can influence their perception of an acceptable quality of life. Additionally, social and cultural factors were not considered in this analysis.

\section{Conclusions}

These data from the 24-month CHOICE study support those from other studies suggesting that PROs are not adversely affected, and may be improved, by the initiation of injectable therapy. As patients are taking a more active role in treatment decisions [3], and as the patient's perception of the effects of their treatment on their quality of life may affect adherence, and therefore clinical effectiveness, PRO data can help the clinician to select the most appropriate treatment for individual patients. We believe that data such as ours enable better understanding of the psychological, as well as clinical, aspects associated with treatment selection in routine clinical practice and will become of increasing importance in shared clinical decisionmaking. Further research is needed to better understand psychosocial aspects that affect how patients value health and treatments [13] and to identify the most important PRO measures from an economic point of view, as well as those most associated with clinical improvements [49].

\section{Competing interests}

Matthew Reaney, Jacek Kiljański, Carole Salaun-Martin, and Hélène Sapin are employees of Eli Lilly: Jacek Kiljański and Carole Salaun-Martin are holders of Eli Lilly shares and share options; Matthew Reaney and Hélène Sapin are not shareholders.

Chantal Mathieu is an advisory board member for Lilly Belgium. Thure Krarup is an advisory board member for Lilly Denmark.

Stephan Matthaei and Bruno Guerci have received honoraria from Eli Lilly for lectures and consultancy.

Claes-Göran Östenson has received honoraria from Eli Lilly for consultancy. Michael Theodorakis has declared that he has no conflicts of interest.

\section{Authors' contributions}

All authors contributed to the study conduct/data collection, analysis, and writing of this manuscript. Matthew Reaney and Jacek Kiljański contributed to the design of the study. All authors read and approved the final manuscript.

Authors' information

Michael Theodorakis: Institute for Clinical Investigation, Bionian Health Sciences Cluster, 5a Stamatas Av, Drossia, GR 145 75, Athens, Greece.

\section{Acknowledgments}

The study and the development of this manuscript were sponsored by Eli Lilly and Amylin Pharmaceuticals, LLC. The authors would like to acknowledge Claire Lavin (Rx Communications, Mold, UK) for medical writing assistance with the preparation of this article, funded by Eli Lilly.

\section{Author details}

${ }^{1}$ Eli Lilly, Windlesham, Surrey, UK. ${ }^{2}$ Department of Endocrinology, UZ Gasthuisberg, Leuven, Belgium. ${ }^{3}$ Department of Molecular Medicine and Surgery, Karolinska Institutet, Stockholm, Sweden. ${ }^{4}$ Diabetes-Center Quakenbrück, Quakenbrück, Germany. ${ }^{5}$ Department of Endocrinology I, Bispebjerg Hospital, Copenhagen, Denmark. ${ }^{6}$ Eli Lilly, Warsaw, Poland. ${ }^{7}$ Eli Lilly, Neuilly Cedex, France. ${ }^{8}$ Department of Clinical Therapeutics, University of Athens School of Medicine, Athens, Greece. ${ }^{9}$ Diabetologie, Maladies Metaboliques \& Nutrition, Hôpital Brabois, CHU de Nancy, et ClC Inserm, ILCV, 54500 Vandoeuvre Lès Nancy, France.

Received: 19 February 2013 Accepted: 2 December 2013 Published: 26 December 2013

\section{References}

1. International Diabetes Federation. IDF Diabetes Atlas: The Global Burden. 6th edition. http://www.idf.org/diabetesatlas.

2. Saydah SH, Fradkin J, Cowie CC: Poor control of risk factors for vascular disease among adults with previously diagnosed diabetes. JAMA 2004, 291:335-342.

3. Inzucchi SE, Bergenstal RM, Buse JB, Diamant M, Ferrannini E, Nauck M, Peters AL, Tsapas A, Wender R, Matthews DR: Management of hyperglycaemia in type 2 diabetes: a patient-centered approach. Position statement of the American diabetes association (ADA) and the European association for the study of diabetes (EASD). Diabetologia 2012, 55:577-596.

4. Holman RR, Paul SK, Bethel MA, Matthews DR, Neil HAW: 10-Year follow-up of intensive glucose control in type 2 diabetes. NEJM 2008, 359:1577-1589.

5. Pfalzgraf AR, Nau DP: The relationship of diabetes symptoms and healthrelated quality of life. Value Health 2001, 4:58. 
6. Hill J, Nielsen M, Fox MH: Understanding the social factors that contribute to diabetes: a means to informing health care and social policies for the chronically ill. Perm J 2013, 17:67-72.

7. Raz I, Riddle MC, Rosenstock J, Buse JB, Inzucchi SE, Home PD, Del Prato S, Ferrannini E, Chan JCN, Leiter LA, LeRoith D, DeFronzo R, Cefalu WT: Personalized management of hyperglycemia in type 2 diabetes. Diabetes Care 2013, 36:1779-1788.

8. Rubin RR, Peyrot M: Quality of life and diabetes. Diabetes Metab Res Rev 1999, 15:205-218.

9. Gulliford MC, Mahabir D: Relationship of health-related quality of life to symptom severity in diabetes mellitus: a study in Trinidad and Tobago. J Clin Epidemiol 1999, 52:773-780.

10. Maddigan SL, Majumdar SR, Toth EL, Feeny DH, Johnson JA, and the DOVE Investigators: Health-related quality of life deficits associated with varying degrees of disease severity in type 2 diabetes. Health Qual Life Outcomes 2003, 1:78.

11. Davies M, Speight J: Patient-reported outcomes in trials of incretin-based therapies in patients with type 2 diabetes mellitus. Diabetes Obes Metab 2012, 14:882-892.

12. Aloumanis K, Benroubi M, Sourmeli S, Drossinos V: Clinical outcomes and costs for patients with type 2 diabetes mellitus initiating insulin therapy in Greece: two-year experience from the INSTIGATE study. Prim Care Diabetes 2013. 10.1016/j.pcd.2013.04.001. [Epub ahead of print].

13. Ali MK, Feeney P, Hire D, Simmons DL, O'Connor PJ, Ganz-Lord F, Goff D Jr, Zhand P, Anderson RT, Narayan KMV, Sullivan MD: Glycaemia and correlates of patient reported outcomes in ACCORD trial participants. Diabet Med 2012, 29:e67-e74.

14. Gönen S, Güngör K, Çilli AS, Kamış Ü, Akpınar Z, Kısakol G, Dikbaş O, Türk S, Hidayetoğlu T, Akça A, Kilinç AC, Kaya A: Comprehensive analysis of health related quality of life in patients with diabetes: a study from Konya Turkey. Turk Jem 2007, 11:81-88.

15. Best JH, Boye KS, Rubin RR, Cao D, Kim TH, Peyrot M: Improved treatment satisfaction and weight-related quality of life with exenatide once weekly or twice daily. Diabet Med 2009, 26:722-728.

16. Best JH, Rubin RR, Peyrot M, Li Y, Yan P, Malloy J, Garrison LP: Weight-related quality of life, health utility, psychological well-being, and satisfaction with exenatide once weekly compared with sitagliptin or pioglitazone after 26 weeks of treatment. Diabetes Care 2011, 34:314-319.

17. Boye KS, Matza LS, Oglesby A, Malley K, Kim S, Hayes RP, Brodows R: Patient-reported outcomes in a trial of exenatide and insulin glargine for the treatment of type 2 diabetes. Health Qual Life Outcome 2006, 4:80.

18. Ware JH, Hamel MB: Pragmatic trials - guides to better patient care? N Engl J Med 2011, 364:1865-1867.

19. Ligthelm RJ, Borzi V, Gumprecht J, Kawamori R, Wenying Y, Valensi P: Importance of observational studies in clinical practice. Clin Ther 2007 29:1284-1292

20. Mann CJ: Observational research methods. Research design II: cohort, cross sectional, and case-control studies. Emerg Med J 2003, 20:54-60.

21. Matthaei S, Reaney M, Mathieu C, Östenson C-G, Krarup T, Guerci B, Kiljanski J, Petto H, Bruhn D, Theodorakis M: Patients with type 2 diabetes initiating exenatide BID or insulin in clinical practice: CHOICE study. Diabetes Ther 2012, 3:6.

22. Mathieu C, Ostenson C-G, Matthaei S, Reaney M, Krarup T, Guerci B, Kiljański J, Salaun-Martin C, Sapin H, Theodorakis M: Using Exenatide twice daily or insulin in clinical practice: results from CHOICE. Diabetes Ther 2013. 10.1007/s133000-013-0037-8. [Epub ahead of print].

23. Östenson C-G, Matthaei S, Reaney M, Krarup T, Guerci B, Kiljanski J, Salaun-Martin C, Sapin H, Bruhn D, Mathieu C, Theodorakis M: Treatment outcomes after initiation of exenatide twice daily or insulin in clinical practice: 12-month results from CHOICE in six European countries. Diabetes Metab Syn Obesity: Targets and Therapy. In press.

24. Kiiskinen U, Matthaei S, Reaney M, Mathieu C, Ostenson C-G, Krarup T, Theodorakis M, Kiljański J, Salaun-Martin C, Sapin H, Guerci B: Resource use and costs of Exenatide BID or insulin in clinical practice: the European CHOICE study. Clin Econ Outcomes Res. In press.

25. Kolotkin RL, Crosby RD, Kosloski KD, Williams GR: Development of a brief measure to assess quality of life in obesity. Obes Res 2001, 9:102-111.

26. Kolotkin RL, Norquist JM, Crosby RD, Suryawanshi S, Teixeira PJ, Heymsfield SB, Erondu N, Nguyen AM: One-year health-related quality of life outcomes in weight loss trial participants: comparison of three measures. Health Qual Life Outcome 2009, 7:53.

27. EuroQol Group: EuroQol-a new facility for the measurement of healthrelated quality of life. Health Policy 1990, 16:199-208.

28. Krabbe P, Weijnen T: Guidelines for analysing and reporting EQ-5D outcomes. In The Measurement and Valuation of Health Status Using EQ-5D: A European Perspective. Edited by Brooks R, Rabin R, de Charro F. Dordrecht: Kluwer Academic Publishers; 2003.

29. Meadows KA, Abrams C, Sandbæk A: Adaptation of the diabetes health profile (DHP-1) for use with patients with type 2 diabetes mellitus: psychometric evaluation and cross-cultural comparison. Diabet Med 2000, 17:572-580.

30. Zigmond AS, Snaith RP: The hospital anxiety and depression scale. Acta Psychiatr Scand 1983, 67:361-370.

31. Jones S, Benroubi M, Castell C, Goday A, Liebl A, Timlin L, Nicolay C, Simpson A, Tynan A: Characteristics of patients with type 2 diabetes mellitus initiating insulin therapy: baseline data from the INSTIGATE study. Curr Med Res Opin 2009, 25:691-700.

32. Data on file.

33. Zinman B, Schmidt WE, Moses A, Lund N, Gough S: Achieving a clinically relevant composite outcome of an $\mathrm{HbA} 1 \mathrm{c}$ of $<7 \%$ without weight gain or hypoglycaemia in type 2 diabetes: a meta-analysis of the liraglutide clinical trial programme. Diabetes Obes Metab 2012, 14:77-82.

34. Jaeschke R, Singer J, Guyatt GH: Measurement of health status: ascertaining the minimal clinical important difference. Control Clin Trials 1989, 10:407-415.

35. Guyatt GH, Osoba D, Wu AW, Wyrwich KW, Norman GR, Clinical Significance Consensus Meeting Group: Methods to explain the clinical significance of health status measures. Mayo Clin Proc 2002, 77:371-383.

36. Barton GR, Sach TH, Doherty M, Avery AJ, Jenkinson C, Muir KR: An assessment of the discriminative ability of the EQ-5D index, SF-6D, and EQ VAS, using sociodemographic factors and clinical conditions. Eur J Health Econ 2008, 9:237-249.

37. Mulhern B, Meadows K: Estimating the minimally important difference (MID) of the diabetes health profile-18 (DHP-18) for type 1 and type 2 diabetes mellitus. Discussion paper 2011. http://eprints.whiterose.ac.uk/43132.

38. Puhan MA, Frey M, Büchi S, Schünemann HJ: The minimal important difference of the hospital anxiety and depression scale in patients with chronic obstructive pulmonary disease. Health Qual Life Outcome 2008, 6:46.

39. Crosby RD, Kolotkin RL, Williams GR: An integrated method to determine meaningful changes in health-related quality of life. J Clin Epidemio/ 2004, 57:1153-1160.

40. Wyrwich KW, Norquist JM, Lenderking WR, Acaster S: Industry Advisory Committee of International Society for Quality of Life Research (ISOQOL): Methods for interpreting change over time in patientreported outcome measures. Qual Life Res 2012. in press [Epub ahead of print].

41. Matza LS, Boye KS, Yurgin N, Brewster-Jordan J, Mannix S, Shorr JM, Barber BL: Utilities and disutilities for type 2 diabetes treatment-related attributes. Qual Life Res 2007, 16:1251-1265.

42. Boye KS, Matza LS, Walter KN, Van Brunt K, Palsgrove AC, Tynan A: Utilities and disutilities for attributes of injectable treatments for type 2 diabetes. Eur J Health Econ 2011, 12:219-230.

43. Bergenstal RM, Garrison LP Jr, Miller L-A, Hou L, Blickensderfer A, Zagar A, Stanley S, Bhargava A, Wade R, Herman WH: Exenatide BID observational study (ExOS):results for primary and secondary endpoints of a prospective research study to evaluate the clinical effectiveness of Exenatide BID use in patients with type 2 diabetes in a real-world setting. Curr Med Res Opin 2011, 27:2335-2342.

44. Grandy S, Fox KM, and for the SHIELD Study Group: Change in health status (EQ-5D) over 5 years among individuals with and without type 2 diabetes mellitus in the SHIELD longitudinal study. Health Qual Life Outcome 2012, 10:99.

45. Bouwman V, Adriaanse MC, van't Riet E, Snoek FJ, Dekker JM, Nijpels G Depression, anxiety and glucose metabolism in the general Dutch population: the new Hoorn study. PLoS One 2010, 5:e9971.

46. Speight J, Reaney MD, Barnard KD: Not all roads lead to Rome-a review of quality of life measurement in adults with diabetes. Diabet Med 2009, 26:315-327.

47. Bradley C, Todd C, Gorton T, Symonds E, Martin A, Plowright R: The development of an individualized questionnaire measure of perceived 
impact of diabetes on quality of life: the ADDQoL. Qual Life Res 1999, 8:79-91.

48. U.S. Food and Drug Administration: Guidance for industry: Patient-reported outcome measures: use in medical product development to support labeling claims. 2009. http://www.fda.gov/downloads/Drugs/

GuidanceComplianceRegulatorylnformation/Guidances/UCM193282.pdf.

49. Leplege A, Hunt S: The problem of quality of life in medicine. JAMA 1997, 278:47.

doi:10.1186/1477-7525-11-217

Cite this article as: Reaney et al:: Patient-reported outcomes among patients using exenatide twice daily or insulin in clinical practice in six European countries: the CHOICE prospective observational study. Health and Quality of Life Outcomes 2013 11:217.

\section{Submit your next manuscript to BioMed Central and take full advantage of:}

- Convenient online submission

- Thorough peer review

- No space constraints or color figure charges

- Immediate publication on acceptance

- Inclusion in PubMed, CAS, Scopus and Google Scholar

- Research which is freely available for redistribution 